ARTICLE

https://doi.org/10.1038/s41467-019-10902-w

\title{
The genomic landscape of Epstein-Barr virus- associated pulmonary lymphoepithelioma-like carcinoma
}

Shaodong Hong1,2,3,11, Dongbing Liu (1) 4,5,11, Shuzhen Luo 4,5,11, Wenfeng Fang 1,2,3,11, Jianhua Zhan 1,2,3, Sha Fu 6,7, Yaxiong Zhang ${ }^{1,2,3}$, Xuan Wu ${ }^{1,8}$, Huaqiang Zhou (1) 1,2,3, Xi Chen 1,2,3, Gang Chen 1,2,3, Zhonghan Zhang ${ }^{1,2,3}$, Qiufan Zheng (1) 1,2,3, Xiaobo Li ${ }^{4,5,9}$, Jinghao Chen 4,5,9, Xingmin Liu ${ }^{4}$, Mengyue Lei ${ }^{4,5}$, Chen Ye ${ }^{4,5}$, Jian Wang ${ }^{4,5}$, Huanming Yang 4,5, Xun Xu (D) 4,5, Shida Zhu 4,5, Yunpeng Yang1,2,3, Yuanyuan Zhao 1,2,3, Ningning Zhou ${ }^{1,2,3}$, Hongyun Zhao ${ }^{1,2,3}$, Yan Huang ${ }^{1,2,3}$, Lanjun Zhang ${ }^{2,3,10}$, Kui Wu ${ }^{4,5}$ \& Li Zhang ${ }^{1,2,3}$

Pulmonary lymphoepithelioma-like carcinoma (LELC) is a rare and distinct subtype of primary lung cancer characterized by Epstein-Barr virus (EBV) infection. Herein, we reported the mutational landscape of pulmonary LELC using whole-exome sequencing, targeted deep sequencing and single-nucleotide polymorphism arrays. We identify a low degree of somatic mutation but widespread existence of copy number variations. We reveal predominant signature 2 mutations and frequent loss of type I interferon genes that are involved in the hostvirus counteraction. Integrated analysis shows enrichment of genetic lesions affecting several critical pathways, including NF-kB, JAK/STAT, and cell cycle. Notably, multi-dimensional comparison unveils that pulmonary LELC resemble NPC but are clearly different from other lung cancers, natural killer/T-cell lymphoma or EBV-related gastric cancer in terms of genetic features. In all, our study illustrates a distinct genomic landscape of pulmonary LELC and provides a road map to facilitate genome-guided personalized treatment.

\footnotetext{
${ }^{1}$ Department of Medical Oncology, Sun Yat-sen University Cancer Center, 510060 Guangzhou, China. ${ }^{2}$ State Key Laboratory of Oncology in South China, 510060 Guangzhou, China. ${ }^{3}$ Collaborative Innovation Center for Cancer Medicine, 510060 Guangzhou, China. ${ }^{4}$ BGI-Shenzhen, 518083 Shenzhen, China. ${ }^{5}$ China National GeneBank-Shenzhen, BGI-Shenzhen, 518120 Shenzhen, China. ${ }^{6}$ Department of Pathology, Sun Yat-Sen Memorial Hospital, Sun Yat-Sen University, 510120 Guangzhou, China. ${ }^{7}$ Guangdong Provincial Key Laboratory of Malignant Tumor Epigenetics and Gene Regulation, 510120 Guangzhou, China. ${ }^{8}$ Peking University Shenzhen Hospital, 518036 Shenzhen, China. ${ }^{9}$ BGI Education Center, University of Chinese Academy of Sciences, 518083 Shenzhen, China. ${ }^{10}$ Department of Thoracic Surgery, Sun Yat-sen University Cancer Center, 510060 Guangzhou, China. ${ }^{11}$ These authors contributed equally: Shaodong Hong, Dongbing Liu, Shuzhen Luo, Wenfeng Fang. Correspondence and requests for materials should be addressed to K.W. (email: wukui@genomics.cn) or to L.Z. (email: zhangli6@mail.sysu.edu.cn)
} 
D ulmonary lymphoepithelioma-like carcinoma (LELC) is a rare subtype of primary lung cancer that histologically resembles undifferentiated nasopharyngeal carcinoma $(\mathrm{NPC})^{1}$. First reported in 1987, pulmonary LELC has been recognized to be closely related to Epstein-Barr virus (EBV) infection ${ }^{2,3}$. In the 2015 World Health Organization (WHO) Classification of Lung Tumors, pulmonary LELC was moved from large cell carcinoma to other and unclassified carcinomas ${ }^{4}$. Compared with other types of lung cancers, pulmonary LELC has distinct clinicopathological features. It preferentially affects Asian non-smokers of younger age and has intensive tumor infiltrating immune cells $s^{5}$. Driver mutations such as epidermal growth factor receptor (EGFR) mutation and anaplastic lymphoma kinase $(A L K)$ rearrangement are rarely detected in pulmonary LELC . Palliative chemotherapy has been the major approach for metastatic disease, though immunotherapy singe-agent or in combination with chemotherapy might also be effective in patients with pulmonary LELC, which is currently categorized as non-smallcell lung carcinoma ${ }^{7-9}$. Due to the lack on the genomic data to date, the pathogenesis, rational histology classification and optimal treatment of pulmonary LELC remains not fully defined. Novel therapeutic agents are urgently needed to further improve of the survival of patients suffering from this lethal disease.

Based on the pathological characteristics of pulmonary LELC and its strong association with EBV infection, we hypothesize that pulmonary LELC is distinct from other types of lung cancers but similar to NPC in terms of genomic aberrations. In this study, we report the genomic landscape of a large cohort of pulmonary LELC by whole-exome sequencing (WES, $n=30$ ), targeted deep sequencing (TDS, $n=61$ ), and single-nucleotide polymorphism (SNP) array analysis $(n=46)$ (Supplementary Fig. 2a). We comprehensively compared the genomic profiles of pulmonary LELC with other relevant cancer types to further enhance the understanding of this unclassified carcinomas of the lung. Here, we identify key genetic lesions affecting pulmonary LELC. Integrative analyses through genomic data furthermore highlight pathways that may play critical roles in driving tumor progression. Multidimensional comparative study shows that pulmonary LELC is distinct from other primary lung cancers but share similarities with NPC, in terms of genomic features. These data provide important insights into the pathogenesis of pulmonary LELC and a road map to inform genome-guided personalized treatment for patients suffering from this rare tumor.

\section{Results}

Clinicopathological features. Paired fresh-frozen tumor tissue and adjacent normal tissue, and formalin-fixed, paraffinembedded (FFPE) tissues were collected from a large cohort of 91 pathologically confirmed pulmonary LELC patients (Supplementary Figs. 1 and 2a, Supplementary Data 1 and 2). Among the patients, 43 (47\%) were male, 17 (19\%) had a history of smoking and the median age at diagnosis was 52.5 years (range: 27-71). The majority $(56 \%)$ of the patients were diagnosed at an early stage (stage I, 31 patients; stage II, 21 patients) whose disease-free survival (DFS) and overall survival (OS) were significantly better than those at stage III-IV (Supplementary Fig. 2b).

Somatic aberrations of pulmonary LELC. We analyzed the WES data of 30 cases with a mean coverage of $100 \times$ (discovery cohort; Supplementary Data 3, Supplementary Fig. 3a) and identified 1461 somatic mutations including 1055 non-silent, 392 silent and 14 short insertions and deletions (Fig. 1a and Supplementary Data 4), revealing a low mutation rate (median: 1.2 mutations per megabase [Mb]; Supplementary Fig. 4). The predominant somatic mutation type was $\mathrm{C}: \mathrm{G}>\mathrm{T}: \mathrm{A}$ transitions and $\mathrm{C}: \mathrm{G}>\mathrm{G}: \mathrm{C}$ transversions (Supplementary Fig. 5). Two independent and stable mutational signatures were then identified (Fig. $1 \mathrm{~b}$ and Supplementary Fig. 6). Signature 1 was reported to be positively correlated with age and was universally present in numerous cancer types ${ }^{10}$. Signature 2 was characterized primarily by $\mathrm{C}>\mathrm{T}$ and $\mathrm{C}>\mathrm{G}$ mutations at $\mathrm{TpCpN}$ trinucleotides and was attributed to the overactivity of the AID/APOBEC family of cytidine deaminases. The APOBEC family of proteins play important roles in the innate immune response against virus infections by modification of viral genome ${ }^{11,12}$, although they also might serve as endogenous carcinogenic mutagens ${ }^{13}$. These data imply that the overactivity of APOBEC family genes may be induced in response to EBV infection and participate in the tumorigenesis of pulmonary LELC.

To verify the mutations identified in discovery cohort and to better define the mutation patterns of pulmonary LELC, we performed TDS on a panel of 114 selected genes (Supplementary Fig. 7a) for the 29 cases from discovery cohort and a validation cohort of additional 61 cases with a mean coverage of $300 \times$ and 170x, respectively (Supplementary Fig. 3b, c and Supplementary Data 3). Cross comparison of somatic mutations in discovery cohort showed that $99 \%$ of candidate mutations in WES were confirmed in TDS with a high consistency (0.89) of mutation frequency (Supplementary Fig. 7b and Supplementary Data 5). Combining the data from both cohorts ( $n=91$ subjects), we discovered that 19 genes were affected by non-silent mutations in at least three patients (Fig. 1c and Supplementary Data 4, 6, and 7). Among them, genes with a prevalence of at least $5 \%$ included four tumor suppressor genes previously implicated in cancer (TP53, NOTCH1, MGA, and PTPRD), one negative regulator of NF- $\kappa B$ pathway (TRAF3), one epigenetic modifier $(K M T 2 C)$, and one laminin subunit essential for basement membrane (LAMA4). TP53, TRAF3, MGA, PTPRD, and KMT2C were predicted to be mutually exclusive by MEGSA (corrected $P=0.005$; Supplementary Fig. $8 \mathrm{a})^{14}$, indicating their independent contributions to the carcinogenesis of pulmonary LELC. Although TP53 mutation was the most frequent in our cohort and twice the frequency of previously reported in pulmonary $\operatorname{LELC}^{15}$, it is infrequent as compared to that of other primary lung cancers (Supplementary Fig. 8b). Nevertheless, all the TP53 mutations were located at the structural domains and 93\% (13/14) of these mutations were in the DNA-binding domain (Supplementary Fig. 8c), suggesting the biological consequence of these mutations. Yet, no correlation between TP53 mutation status and patient's survival was observed (Fisher's exact test, $P=0.72$ ). Notably, we observed frequent and mutually exclusive mutations of laminin subunit genes (Fig. 1c and Supplementary Fig. 8d), including LAMA4 (five mutations), LAMA2 (three), and LAMB1 (three). The dysregulation of these laminin subunit genes has been widely reported to promote tumor invasion and metastasis in different cancers ${ }^{16-19}$. These imply that the frequent mutation of laminin subunit genes may also play important roles in the progression of pulmonary LELC and warrant further investigation.

Interestingly, frequently altered driver genes (eg. EGFR, KRAS, and $B R A F$ ) in other lung cancer subtypes were rarely detected in pulmonary LELC, in consistent with previous reports ${ }^{6,20-23}$. Although MET missense mutations were detected in two patients, none of them belong to the canonical MET exon 14 skipping mutations ${ }^{24,25}$. These indicate that typical driver mutations in other lung cancer subtypes do not play a critical role in the carcinogenesis of pulmonary LELC.

Somatic copy number alterations of pulmonary LELC. Somatic copy number alterations (SCNAs) were profiled in 46 tumors with sufficient quantity and quality. The number of genes affected 

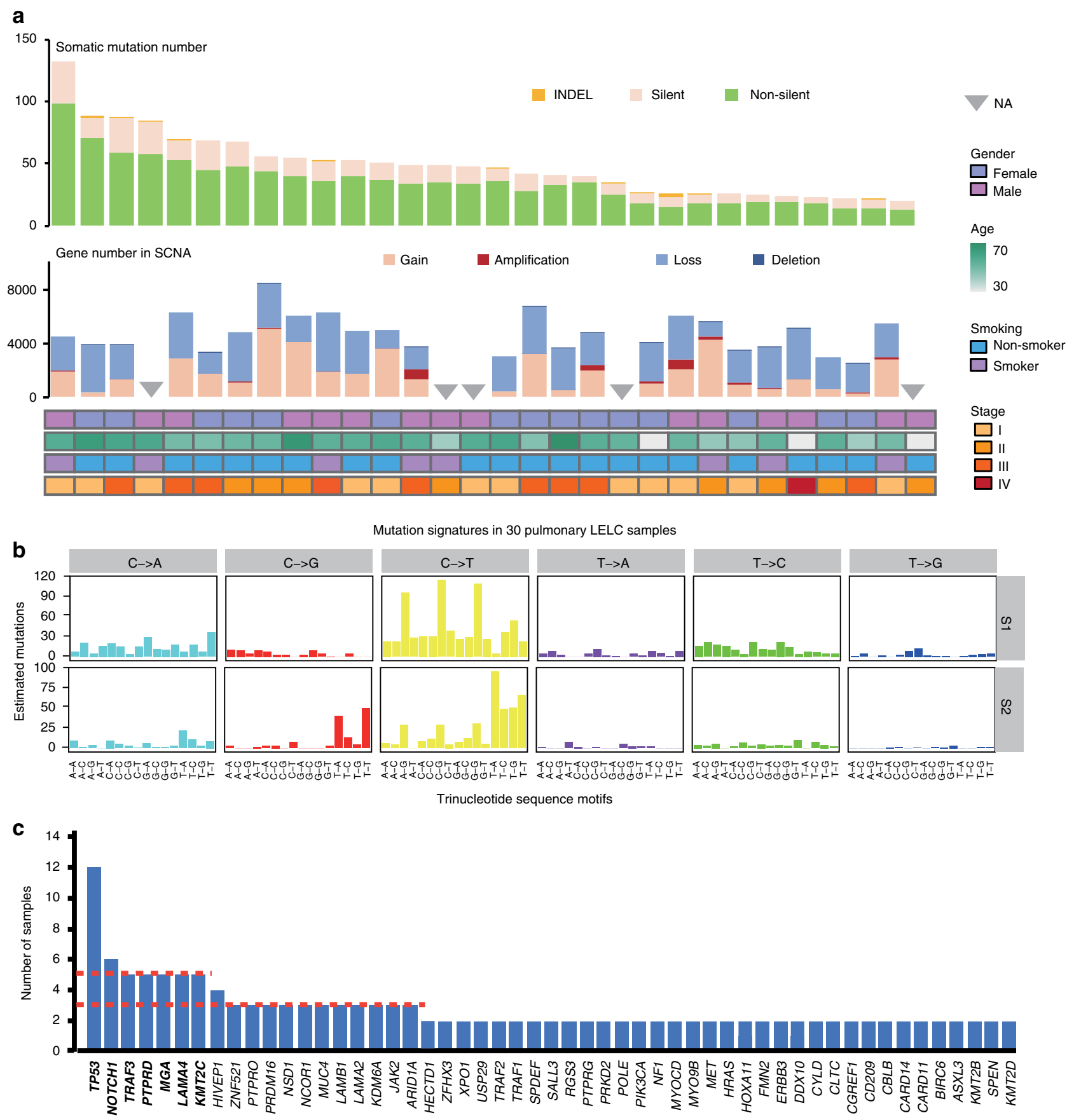

Fig. 1 Somatic mutations and copy number alterations in pulmonary LELC. a The number of somatic mutations and copy number altered genes for each pulmonary LELC samples in the discovery cohort. Gender, age, smoking status, and tumor stages are listed at the bottom according to the samples. b Signatures are displayed according to the 96-substitution classification, with $x$-axis showed mutation types and $y$-axis showed the estimated mutations of each mutation type, which are identified by a Bayesian NMF algorithm. c Frequently mutated genes in the discovery cohort and validation cohort. The two red dashed line denote three and five mutated patients, respectively. Genes mutated in more than five patients are labeled with bold font. LELC, lymphoepithelioma-like carcinoma; SCNA, somatic copy number aberration; NA, not applicable; INDEL, insertion and deletion

ranged from 2381 to 8420 (mean 4600; Fig. 1a and Supplementary Fig. 9a). Frequent arm-level alterations included copy number gains in $5 \mathrm{p}(32 \%), 12 \mathrm{p}(54 \%)$, and $12 \mathrm{q}(48 \%)$ and copy number losses in $3 p(49 \%), 5 q(47 \%), 13 q(36 \%), 14 q(60 \%)$, and 16q (39\%) (Supplementary Fig. 9b). Similar to NPC, frequent losses in $14 \mathrm{q}$ and $16 \mathrm{q}$ were also identified in pulmonary LELC, leading to inactivation of multiple negative regulators of NF- $\mathrm{KB}$ pathway (TRAF3 [14q32.3, 80\%], NFKBIA [14q13, 52\%], NLRC5 [16q13, 52\%], and CYLD [16q12.1, 48\%] $)^{26,27}$. In addition, copy number gain of the whole chromosome 12 was observed in $48 \%$
(22/46) of patients, leading to amplification of 34 cancer related genes annotated in the Catalogue of Somatic Mutations in Cancer (COSMIC) database and signaling pathways including MAPK $(q=0.001)$, JAK/STAT $(q=0.014)$, and cell cycle $(q=0.032)$ (Supplementary Data 8 ). Nineteen significant focal copy number alterations (8 amplifications and 11 deletions) were identified using GISTIC2 (Fig. 2a and Supplementary Data 9) ${ }^{28}$. Significantly amplified regions included 7p11.2, 9p24.1, 11q13.3, and 12p13.2. The predominant event was amplification in 11q13.3, which contained the $C C N D 1$ gene. Amplification of $C C N D 1$ may 
a

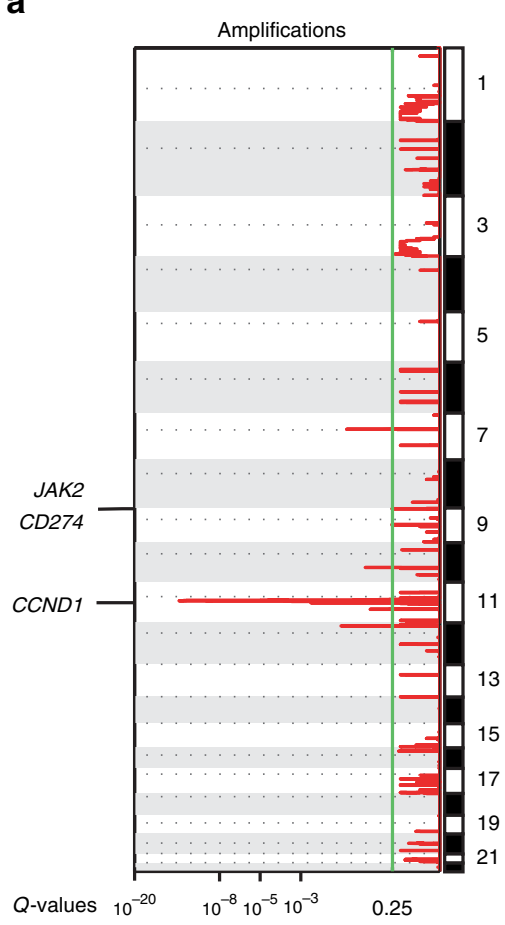

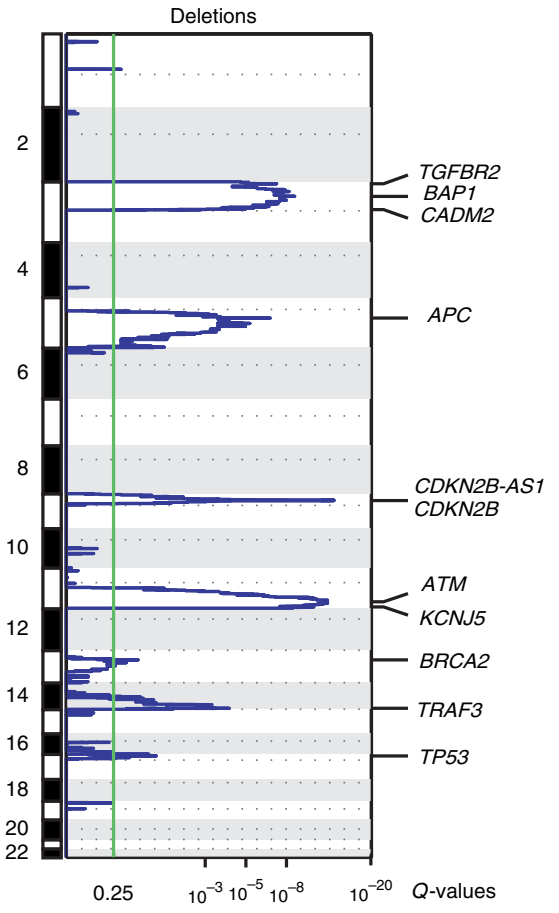
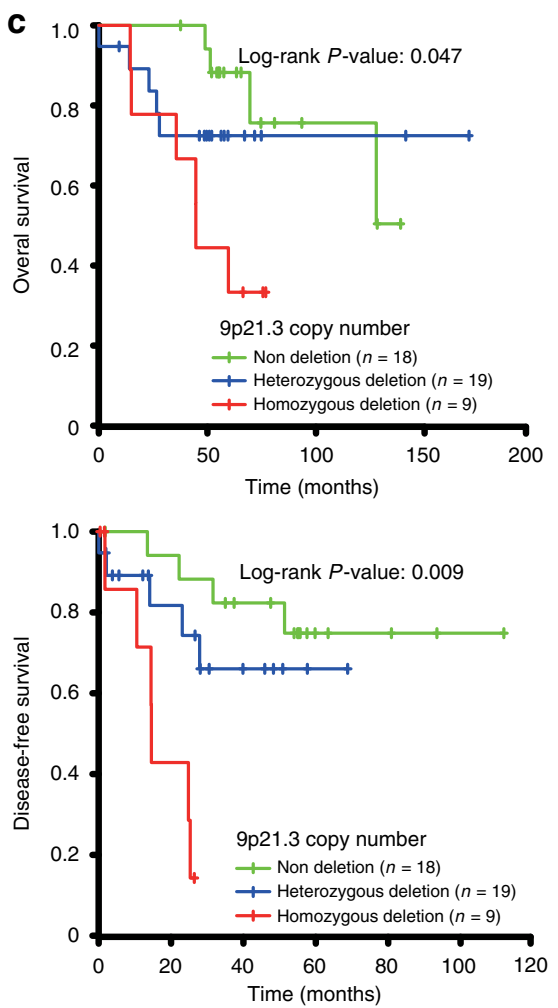

b

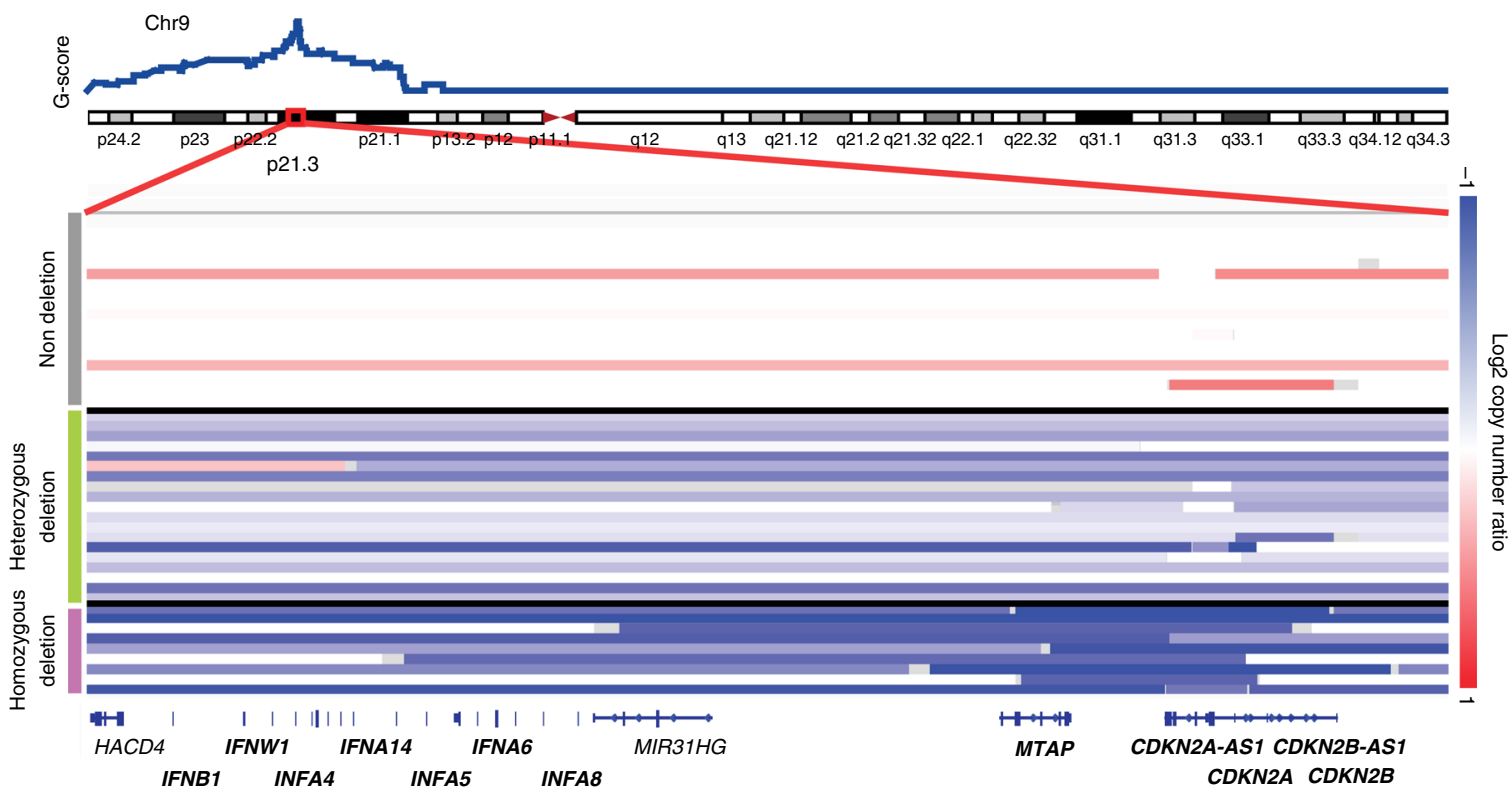

Fig. 2 Analysis of copy number alterations in pulmonary LELC. a Focal amplification and deletion determined from GISTIC 2.0 analysis. The plot shows significant amplification (red) or deletion (blue) for the chromosomes from 1 (top) to 22 (bottom). The green line indicates the cut-off for significance ( $q=0.25$ ). Genes listed on left (for amplifications) and right (for deletions) are likely drivers located in the peak areas defined by GISTIC 2.0. b Zoom in the significant deletion region in p21.3 of chromosome 9. Samples are classified into three groups: samples without deletions; samples with heterozygous deletions; and samples with homozygous deletions. c Kaplan-Meier survival analysis for three groups with different copy number status of 9p21.3. Statistical significance was estimated by two-sided log-rank test. LELC, lymphoepithelioma-like carcinoma

drive cell cycle progression and contribute to tumorigenesis. Furthermore, we found that $C D 274$ was amplified in seven cases (8\%). Amplification of CD274 was associated with elevated programmed cell death ligand-1 (PD-L1) expression in EBVassociated gastric cancer ${ }^{29}$. Indeed, we and others have previously found that PD-L1 was remarkably over-expressed in pulmonary LELC ${ }^{6,23}$. The amplification of CD274 identified herein may provide an alternative mechanism of the overexpression of PD-L1 in pulmonary LELC and the rationale for immunotherapy. Significantly deleted regions included 3p21.31, 3p25.3, 5q14.1, 9p21.3, 11q23.3, 13q14.2, 14q32.32, and 17 p13.1 encompassing a great number of tumor suppressor genes such as BAP1, VHL, APC, ATM, RB1, TRAF3, and TP53 (Fig. 2a and Supplementary Data 9).

Frequent deletion of 9p21.3 was observed in a variety of cancers including NPC and lung cancer ${ }^{30-32}$. In pulmonary 
LELC, a narrow region (chr9:22028316-22041442) of 9p21.3 was also identified as focal and significant deleted by GISTIC2 (Supplementary Data 9). Patients with 9p21.3 focal deletion were significantly associated with poor survival (Fig. 2c). In addition to the focal deletion region, nearby regions within 9 p21.3 also showed high frequency of deletion involving two tumor suppressors (CDKN2A,CDKN2B), MTAP and a cluster of type I IFN genes (Supplementary Data 10, Fig. 2b). The loss of type I IFN genes included all the 13 IFN- $\alpha$ protein coding genes (IFNA1, IFNA2, IFNA4, IFNA5, IFNA6, IFNA7, IFNA8, IFNA10, IFNA13, IFNA14, IFNA16, IFNA17, and IFNA21), IFNE, IFNB1 and IFNW1, affecting a total of 56\% cases (26/46). Biologically, type I IFNs are responsible for the front-line defense against viral infection and are key component in the host-virus standoff ${ }^{33}$. Interestingly, we found that tumors with $9 \mathrm{p} 21.3$ deletion had lower level of CD8 + tumor infiltration lymphocytes (TILs) than those without 9p21.3 deletion, with marginal significance (Student's $t$-test, $P=0.05$; Supplementary Fig. $10 \mathrm{a}$ ). It is hypothesized that frequent loss of type I IFN genes may lead to the defect of host immune response against virus and the persistent EBV infection in pulmonary LELC.

Pathway analysis. Integrated analysis of mutational profiles revealed core signaling pathways implicated in discrete functional categories, including cell cycle, JAK/STAT and NF- $\kappa B$ (Fig. 3). Cell cycle pathway was altered primarily by mutation or deletion of TP53, amplification of MDM2 and CCND1, and deletion of $C D K N 2 A / B$ and $R B 1$, revealing frequent defects in the G1/S transition control (Fig. 3a). JAK/STAT pathway was frequently dysregulated, largely owing to deletion of CISH (in $95 \%$ of the

\section{a}

Cell cycle

Somatic mutation altered: $18 \%$ (12/91); copy number altered: $83 \%$ (38/46)

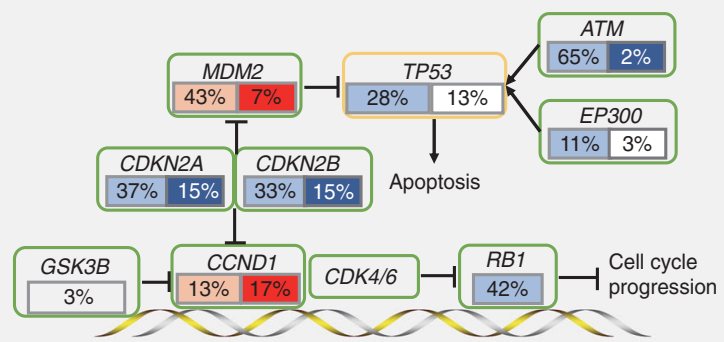

C

NF-kappa B signaling pathway

Somatic mutation altered: 18\% (12/91); copy number altered: 93\% (43/46)

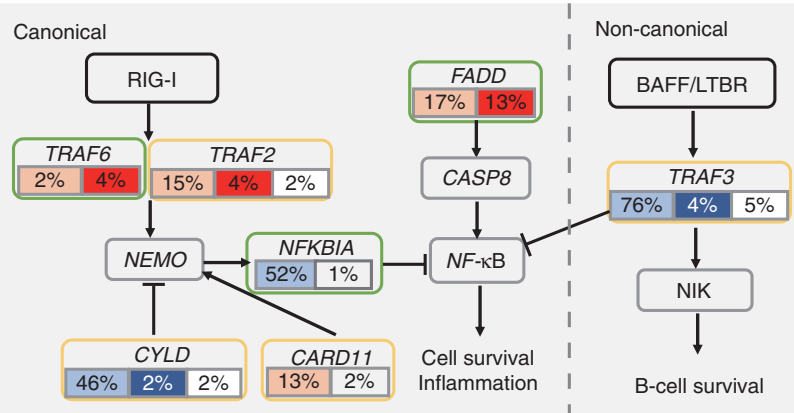

patients), followed by mutation or deletion of $P T P R D$, and mutation or amplification of JAK2 (Fig. 3b). CISH encodes the cytokine-inducible $\mathrm{SH} 2$-containing protein from the suppressors of cytokine signaling (SOCS) family which are the major negative regulators of the JAK/STAT pathway ${ }^{34}$. PTPRD encodes a tumor suppressor that negatively regulates JAK/STAT pathway by dephosphorylating and inactivating STAT3 oncoprotein ${ }^{35}$. JAK2 and its downstream signaling cascade genes such as $P I 3 K C A / B$ were also frequently altered in pulmonary LELC. It is of note that JAK/STAT pathway could be activated by IFNs in response to pathogen invasion and induce the transcription of numerous IFN-stimulated genes (ISGs) ${ }^{36}$. Therefore, the widespread deletion of type I interferon genes discussed above could lead to defects in the IFN-induced JAK/STAT activation and the subsequent anti-viral immune response in pulmonary LELC. NF- $\kappa$ B pathway aberration was implicated in $18 \%$ of patients by somatic mutations and $93 \%$ of patients by SCNAs (Fig. 3c). Negative regulators of NF- $\mathrm{kB}$ pathway including TRAF3, CYLD, NFKBIA, and NLRC5 were frequently deleted in pulmonary LELC. Moreover, recurrent somatic mutations of TRAF3 and CYLD were identified in five and two cases, respectively. Defects in TRAF3/CYLD have been implicated in the activation of NF- $\kappa$ B signaling in HPV-positive head and neck squamous cell carcinoma (HNSCC) and EBV-positive $\mathrm{NPC}^{26,37}$, suggesting that TRAF3 and CYLD genetic alterations may also participate in EBV-mediated tumorigenesis in pulmonary LELC. In addition to deletion of negative regulators, somatic mutations or amplification of multiple components of the canonical NF- $\kappa B$ pathway were also identified, including FADD, TRAF2, TRAF6, and CARD11. FADD is an apoptotic adapter molecule that activates the NF- $\kappa \mathrm{B}$ pathway by recruiting caspase- 8 . TRAF2 and TRAF6 b

Jak/STAT signaling pathway

Somatic mutation altered: 14\% (13/91); copy number altered: 95\% (44/46)
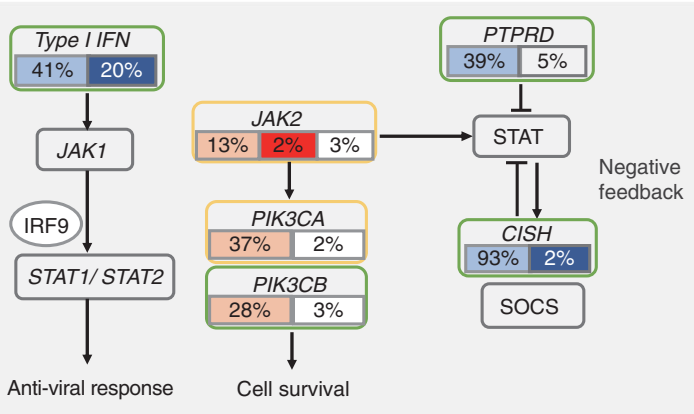

Fig. 3 Altered pathways in pulmonary LELC. Alterations defined as somatic mutations, focal amplifications, and deletions affecting Cell cycle (a), JAK/ STAT (b), and NF-kappa B (c) signaling pathways are shown. Alteration frequencies are expressed as a percentage of samples form discovery cohort and validation cohort. LELC, lymphoepithelioma-like carcinoma 
are two members of the TNF receptor associated factor (TRAF) protein family, which mediate activation of NF- $\kappa$ B pathway and are involved in the regulation of inflammation, antiviral responses, and apoptosis ${ }^{38}$. CARD11 encodes the caspase recruitment domain-containing protein 11 that functions as a positive regulator of NF- $\kappa \mathrm{B}$ activation by interacting with and inducing phosphorylation of BCL10 39,40 .

Pathway aberrations are mutually exclusive with LMP1. LMP1 is a viral oncoprotein that potentially activates the NF-kB and JAK/STAT pathways, and promotes cell cycle progression in $\mathrm{NPC}^{41-43}$. High LMP1 expression was detected in $19(20.9 \%)$ of 91 pulmonary LELC cases by immunohistochemistry, similar to that in $\mathrm{NPC}^{44}$. We also identified mutual exclusivity among LMP1 overexpression and the three core pathway aberrations (Supplementary Data 11) including NF-kB (Fisher's exact test, $P=0.00005$; Supplementary Fig. 11a), JAK/STAT (Fisher's exact test, $P=0.00934$; Supplementary Fig. 11b) and cell cycle (Fisher's exact test, $P=0.00954$; Supplementary Fig. 11c). These data support the hypothesis that somatic genetic alterations and viralmediated events synergistically participated in the carcinogenesis of pulmonary LELC.

Immune microenvironment in relation to genomic alterations. Immunostaining for PD-L1 was observed in the membrane and/ or cytoplasm of the tumor cells and stromal lymphocytes. We restricted our analysis to CD8 positive TILs due to the fact that these cells are generally thought to be the main effector population following treatment with immune checkpoint inhibitors. Representative PD-L1 and CD8 staining is shown in Supplementary Fig. 1. We found that tumors with 9p24.1 amplification (where CD247 gene located) was significantly associated with higher PD-L1 expression than those without 9p24.1 amplification (Student's $t$-test, $P=0.01$; Supplementary Fig. 10b). However, there were no significant associations between PD-L1 overexpression and number of signature 2 mutations, percentage of EBV type 1 reads, somatic mutation burden or the three core signaling pathways (Supplementary Fig. 10c). Also, no significant correlation was observed between CD8 + TILs and number of signature 2 mutations, percentage of EBV type 1 reads, somatic mutation burden or the three core signaling pathways (Supplementary Fig. 10c).

TRAF3 is a tumor suppressor in pulmonary LELC. TRAF3 functions as a negative regulator of the non-canonical NF- $\kappa$ B pathway ${ }^{45}$. It can also interact with EBV-encoded latent infection membrane protein-1 (LMP1), which may be essential for the oncogenic effects of LMP1 in $\mathrm{NPC}^{41}$. In our pulmonary LELC cohort, TRAF3 mutation was identified in five cases (Fig. 1c and Supplementary Data 4 and 6). Among them, one was nonsense mutation that results in truncated protein product and the other four mutations affected highly conserved residues (Fig. 4a), suggesting that these mutations may alter the protein function and are biologically consequential. Furthermore, TRAF3 deletion was ubiquitously observed in pulmonary LELC (80\%) (Fig. 4b). Given the frequent TRAF3 aberrations in pulmonary LELC, we further examined its biological function in BEAS-2B cells. Knockdown of wild-type endogenous TRAF3 expression with short hairpin RNAs (shRNAs) up-regulated key components of NF- $\kappa \mathrm{B}$ signaling pathway including p52 and IK $\beta a$ (Fig. 4c) and led to markedly increased cell growth, cell migration, and colony formation (Fig. 4d-f). These findings indicate that TRAF3 functions as a tumor suppressor gene and may participate in the tumorigenesis of pulmonary LELC.
Comparison with other lung cancers and NPC. Given the ambiguous classification of pulmonary LELC, we comprehensively compared its genetic features with lung adenocarcinoma (LUAD), lung squamous cell carcinoma (LUSC), small-cell-lung carcinoma (SCLC), NPC, natural killer/T-cell lymphoma (NKTCL), EBV-positive and EBV-negative stomach cancer, and HNSCC. Analysis of mutation rate, mutation spectrum of six substitution categories or hierarchical clustering based on 96 trinucleotide mutational contexts demonstrated that pulmonary LELC resembled NPC and NKTCL but was clearly different from other lung cancers (Fig. 5a-c and Supplementary Fig. 12a, b). All lung cancer subtypes except pulmonary LELC were characterized by frequent $C: G>A: T$ transversions of tobacco smoking fingerprint. Moreover, clustering by the mutation frequency of significantly altered genes revealed that other lung cancers had broad spectra of mutated genes, whereas pulmonary LELC, NPC, and NKTCL harbored rare gene mutations (Supplementary Fig. 12c). To avoid the bias caused by the low mutation rate of EBV-positive cancers, we carried out SCNAs comparison (Fig. 5d and Supplementary Fig. 13a). Again, we found pulmonary LELC had very different SCNAs landscape from other lung cancers. LUAD, LUSC, and SCLC showed apparent amplifications of $1 \mathrm{q}, 5 \mathrm{p}, 8 \mathrm{q}$ and deletion of $8 \mathrm{p}$, which were absent in pulmonary LELC. Notably, NKTCL showed less SCNAs than pulmonary LELC and NPC did and had an evident higher propensity for deletion of $6 \mathrm{q}$, consistent with previous studies $^{46,47}$. Both pulmonary LELC and NPC showed amplification of the whole chromosome 12 and deletion of $3 p$ (contained BAP1), 13q (RB1), 14q (TRAF3), and 16q (CYLD). Hierarchical clustering by SCNAs confirmed that pulmonary LELC was grouped with NPC (Supplementary Fig. 13b). Next, we compared the frequency of SCNAs in genes involved in three major pathways (Fig. 5e). We found that pulmonary LELC shared similar altered frequency for almost all the evaluated pathway genes with NPC, particularly NF- $\kappa$ B. Finally, we found similar abundance of EBV sequences between pulmonary LELC and NPC in the WES data, which was much higher than that of NKTCL (Supplementary Fig. 14).

Gemcitabine has better antitumor activity than pemetrexed. Gemcitabine plus cisplatin has been demonstrated to improve survival of metastatic or recurrent NPC compared to fluorouracil plus cisplatin; while the efficacy of pemetrexed for NPC was very limited ${ }^{48-50}$. As pulmonary LELC resembles NPC in terms of genetic and histopathological features but was currently classified as non-squamous cell lung carcinoma, we retrospectively evaluated the efficacy of gemcitabine plus platinum (GP, $n=21$ ) vs. pemetrexed plus platinum (AP, $n=38$ ) as firstline treatment for metastatic pulmonary LELC. Among the patients, 27 (46\%) were male, 19 (32\%) had a history of smoking and the median age at diagnosis was 49 years (range: 29-74). Baseline characteristics of this cohort are presented in Supplementary Data 12. The results showed that GP significantly improved objective response rate $(76.19 \%$ vs. $23.68 \%$; Pearson's $\chi^{2}$ test, $P<0.001$ ) and progression-free survival (median 8.80 vs. 6.53 months; Log-rank test, $P=0.009$ ) compared to AP (Supplementary Fig. 15). In multivariate analysis controlling for potential confounding factors including age, gender, performance status, stage, and number of metastatic organs, gemcitabine plus cisplatin remained significantly associated with improved progression-free survival (Cox proportional-hazards regression, $P=0.024$ ) and overall response (Cox proportionalhazards regression, $P=0.001)$ compared with pemetrexed plus cisplatin (Supplementary Data 13). 
a

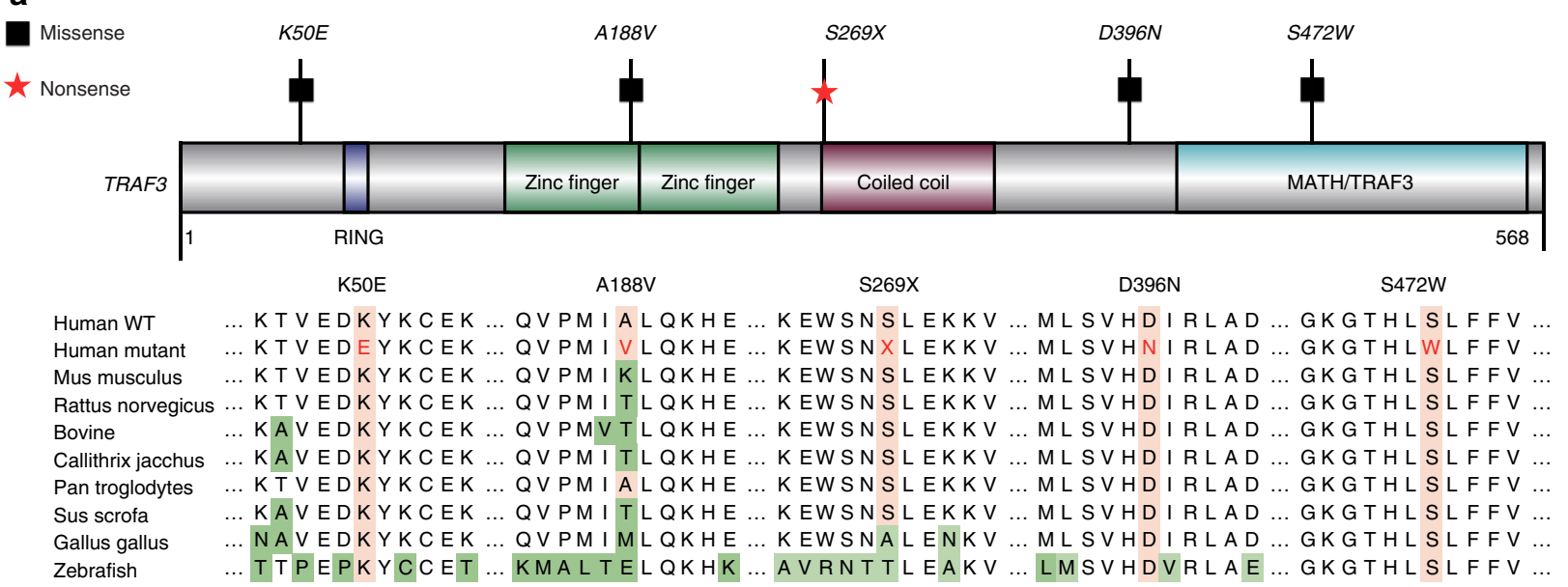

b
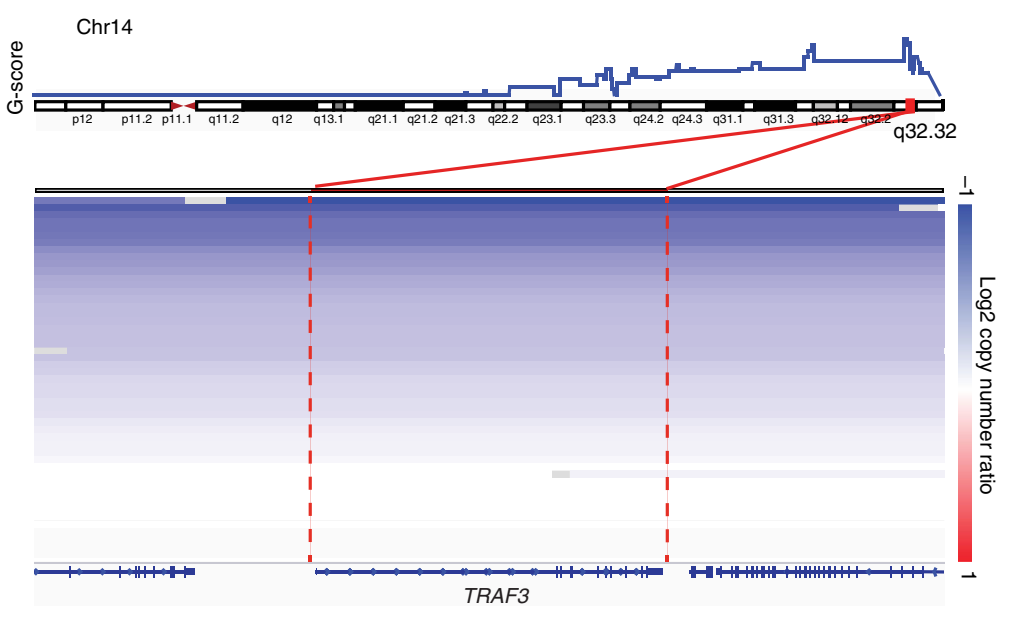

d

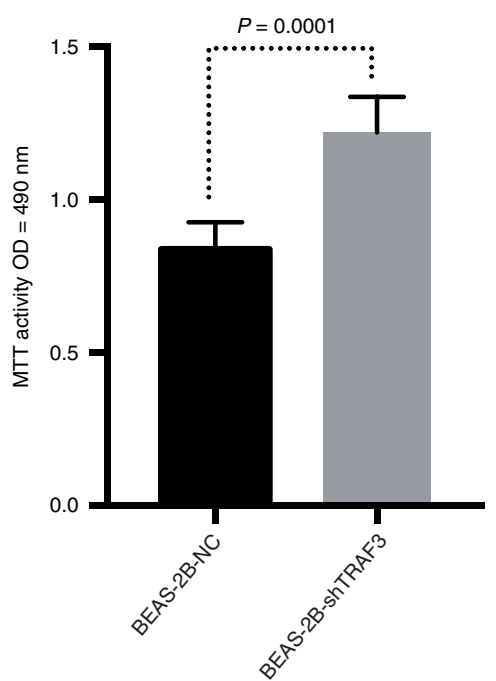

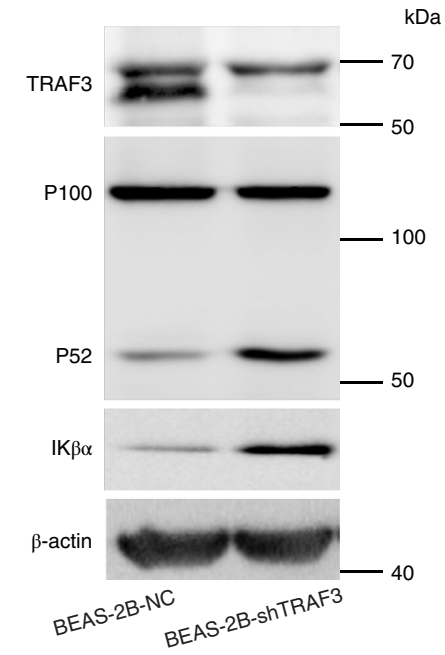

f

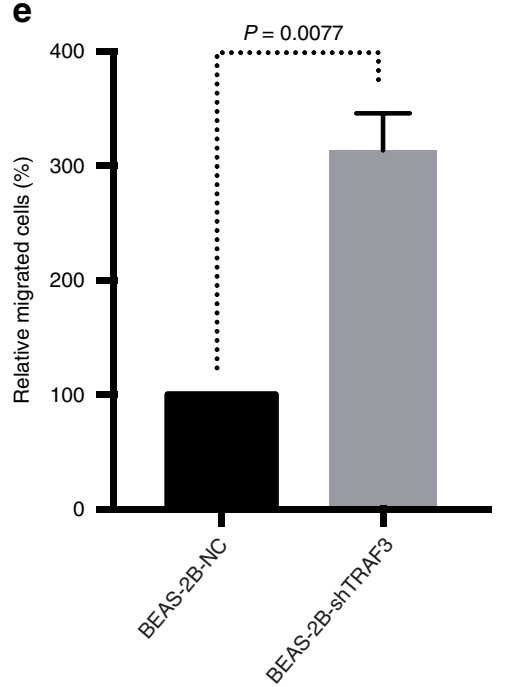

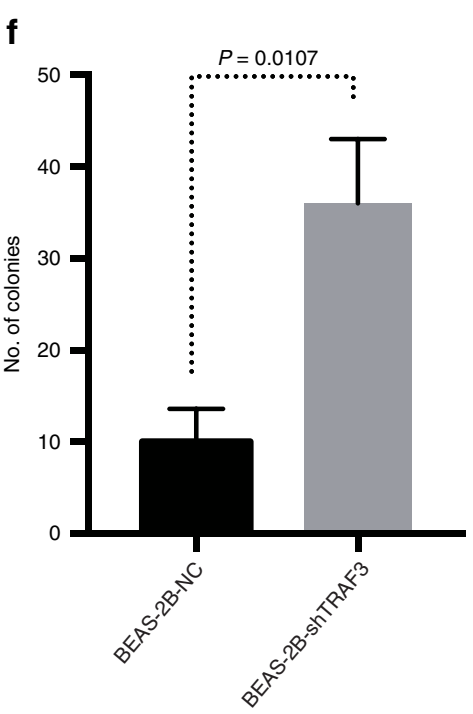

Fig. 4 TRAF3 alterations in pulmonary LELC. a Protein domain structure of TRAF3 based on UniProt database with mutated sites. Sequence alignment of TRAF3 protein across distinct species is shown. Amino acid positions of the mutations are indicated above the alignment. $\mathbf{b}$ Copy number deletions of TRAF3, samples are sorted by log2 copy number ratio. BEAS-2B cells either with or without knockdown of endogenous TRAF3 expression with short hairpin RNAs (shRNAs) were examined by western blot analysis (c), MTT assay (d), migration assay (e), and colony formation assay (f). Error bars in $\mathbf{d}, \mathbf{e}$, and $\mathbf{f}$ denote standard error of the mean. Experiments for $\mathbf{d}, \mathbf{e}$, and $\mathbf{f}$ were performed in triplicate. Student's $t$-test was used for statistical analysis of d, e, and f. NC, normal control; MTT, 3-(4, 5-dimethylthiazol-2-yl)-2, 5-diphenyltetrazolium bromide; OD, optical density 


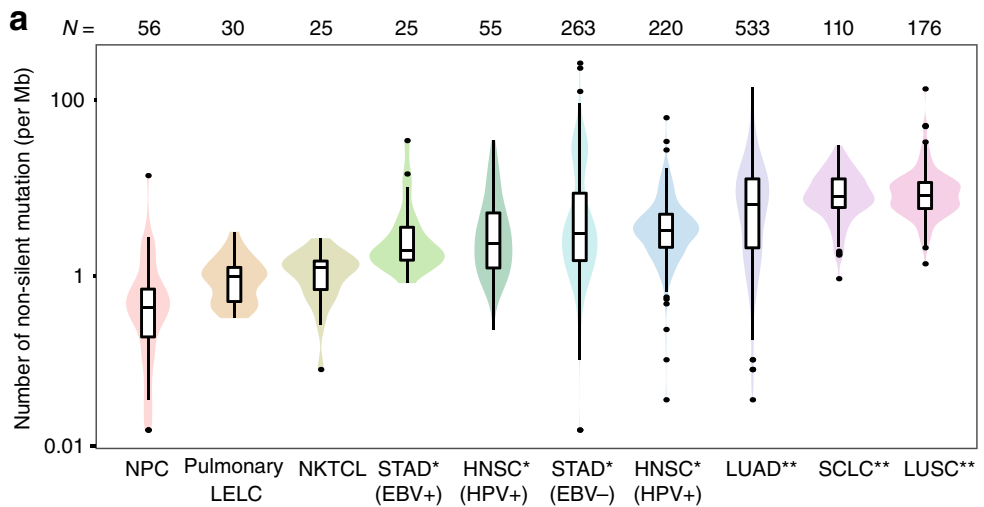

C
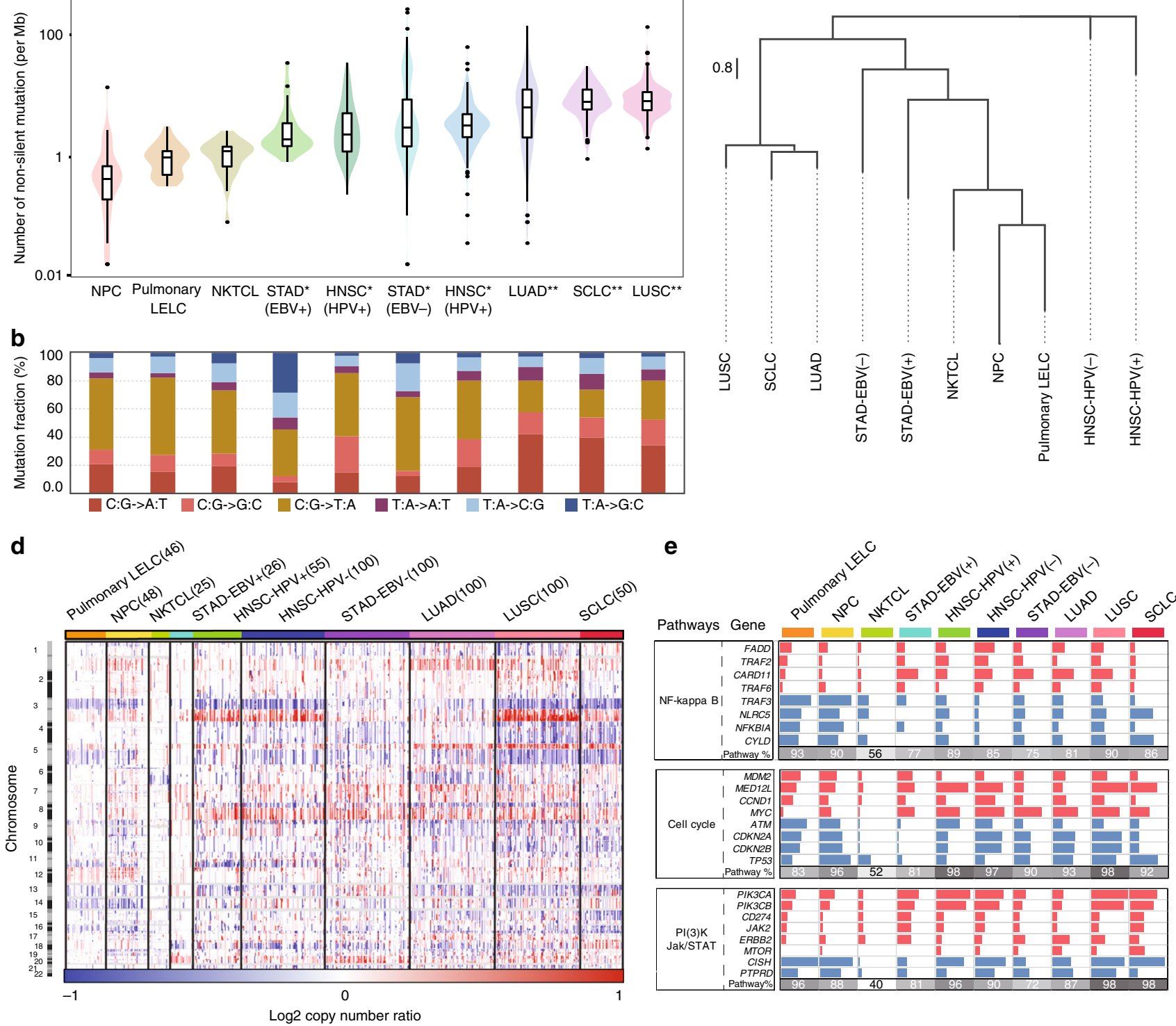

Fig. 5 Comparison between pulmonary LELC and other associated cancer types. a Distribution of non-silent mutation rates of pulmonary LELC and other associated cancer types. The upper numbers indicate the number of samples for each cancer type. Black lines in the boxplot denote median mutation rate for each cancer type and outliers are shown as dots. All the other cancer types are statistically tested by unpaired two-side $t$-test with pulmonary LELC. ${ }^{\star} P$-value $<0.05$ and ${ }^{\star \star} P$-value $<0.0001$. b Mutation spectrum of six mutation type for each cancer type. c Clustering of 96 subtypes based on six mutation types and nucleotides flanking the mutated base for each cancer type. d SCNA comparison of pulmonary LELC and other cancer types. e Frequency comparison of genes with copy number amplification (red) or deletion (blue) for three major oncogenic pathways: NF-kappa B, Cell cycle and Jak/STAT/PI (3)K. Percentages of samples mutated in each cancer type are shown in gray. NPC, nasopharyngeal carcinoma; LELC, lymphoepithelioma-like carcinoma; NKTCL, natural killer/T cell lymphoma; STAD, stomach adenocarcinoma; HNSC, head and neck squamous cell carcinoma; EBV, Epstein-Barr virus; HPV, human papillomavirus; LUAD, lung adenocarcinoma; SCLC, small-cell lung carcinoma; LUSC, lung squamous cell carcinoma; SCNA, somatic copy-number alterations; $\mathrm{Mb}$, megabase

\section{Discussion}

In summary, our study of 91 pulmonary LELCs using WES, TDS, SNP arrays analysis and functional experiments revealed the distinct mutational landscape of this special subtype of lung cancer. We identified an infrequent somatic mutation rate but the widespread existence of copy number alterations in pulmonary LELC. We also discovered novel genomic events affecting several key pathways that might contribute to the tumorigenesis of this disease, capital among these being cell cycle, JAK/STAT and NF$\kappa \mathrm{B}$. The involvement of viral infection in pulmonary LELC pathogenesis was also demonstrated in the study. More importantly, by multidimensional genomic comparison, we unveiled that pulmonary LELC is a unique subtype of lung cancer that genetically resembles NPC.

Given the rarity of somatic driver mutations in pulmonary LELC and the fact that this disease is closely related to EBV infection, the underlying mechanism of tumorigenesis is of special interest. In this study, we detected positive EBV-encoded RNA (EBER) staining by in situ hybridization (ISH) and EBV sequence by WES for all the tumor cases, confirming the presence of EBV infection in pulmonary LELC. Besides, we identified widespread signature 2 mutations, which are attributed to the overactivity of AID/APOBEC family of cytidine deaminases that participate in the antiviral innate immune response partially 
through inducing transcription of type I interferons, as well as modification of viral genome. However, accumulating evidence also suggests that APOBEC family proteins could on the other hand serve as endogenous mutagens for carcinogenesis. Furthermore, the frequently dysregulated NF- $\kappa$ B pathway could be hijacked by the invading viruses to prolong survival of the host cell in order to buy time for viral replication and progeny production $^{51}$. Notably, we also identified ubiquitous losses of type I IFN genes in pulmonary LELC, which led to defect in the production of anti-viral cytokines and IFN-dependent JAK/STAT activation. Previous studies showed that type I interferon might enhance CD8 $+\mathrm{T}$ cell effector function, systematically activate natural killer $(\mathrm{NK})$ cell activity and increase antigen presentation of the tumor cells to be recognized by $\mathrm{T}$ lymphocytes ${ }^{52-54}$. Therefore, it might be inferred that the frequent loss of type I interferon genes may impair the efficacy of immune checkpoint inhibitor therapy. Collectively, the APOBEC family gene signature, dysregulated NF- $\mathrm{B}$ pathway and loss of type I IFN genes are likely responsible for the EBV-induced carcinogenesis of pulmonary LELC and might facilitate development of novel therapeutic strategies.

We also found that TRAF3 was ubiquitously altered in pulmonary LELC, including $5 \%$ of simple somatic mutation and $80 \%$ of deletion. Functional experiments confirmed that TRAF3 served as a tumor suppressor gene and negatively regulated NF- $\kappa B$ pathway. Therefore, TRAF3 loss accounts for the core element of $\mathrm{NF}-\kappa \mathrm{B}$ dysregulation and plays important role in tumorigenesis of pulmonary LELC. It could be conceived of that NF-kB inhibitors can potentially be used as novel therapeutics in pulmonary LELC patients.

Finally, besides the known histological similarity with NPC, we provided the first comprehensive genetic landscape comparison between pulmonary LELC and NPC, as well as other primary lung cancers. We revealed clear difference of mutation spectrum, significant somatic mutations, copy number alterations, and signaling pathway aberrations between pulmonary LELC and other lung cancers; whereas, pulmonary LELC resembles NPC genetically, e.g., low degree of somatic mutation, exclusivity of LMP1 overexpression with somatically altered signaling pathways, frequent chromosomal alterations and predominant NF- $\kappa \mathrm{B}$ dysregulation. In addition, we showed that gemcitabine might improve response rate and progression-free survival compared with pemetrexed as first-line palliative chemotherapy in metastatic pulmonary LELC. This preliminary clinical data may also add evidence for the similarity between this unique lung cancer and NPC.

One major limitation of the current study is that the methods we applied could not provide other genomic information such as chromosomal alterations and transcriptome. Further studies are needed to fully unveil the genomic architecture of pulmonary LELC. Secondary, because this study focused on the genomic features of this special tumor, we did not dig into the exact viral integration sites or the detailed mechanisms of EBV-induced carcinogenesis. However, our study did have provided important insights into further research in this area.

In conclusion, our study delineated a comprehensive view of genomic alterations in pulmonary LELC, defined potential mechanism of tumorigenesis and provided evidence that pulmonary LELC is a distinct lung cancer that resembles NPC. The data presented here might offer novel avenues for treatment of this lethal malignancy and are important for future revision of histological classification of lung tumors.

\footnotetext{
Methods

Patients and samples. The study retrospectively collected fresh-frozen tumor tissue and matched tumor adjacent normal tissue as well as Formalin-fixed,
}

Paraffin-embedded (FFPE) tissue from 91 pulmonary LELC patients for genomic characterization. All the patients had surgical resection in Sun Yat-sen University Cancer Center (SYSUCC) between April 2002 and April 2014 (Supplementary Data 1 and 2). An additional cohort of 59 metastatic pulmonary LELC who received first-line palliative chemotherapy between April 2011 and September 2017 were retrospectively included for further survival analysis (Supplementary Data 12). The primary endpoints of the second cohort analysis were objective response rate and progression-free survival between two treatment groups. Nasopharyngoscopy or Magnetic Resonance Imaging (MRI) was done to rule out lung metastasis from NPC in all the patients. Pathological diagnoses were established according to the WHO classification and independently reviewed by two pathologists. All tumor cases were confirmed to be positive for EBV-encoded RNA (EBER) staining determined using the EBV Probe In Situ Hybridization (ISH) Kit (Triplex International Biosciences, China). For the genomic study, proportion of tumor content must be $30 \%$ or more (Supplementary Data 1). Detailed clinical characteristics were summarized in Supplementary Data 1,2, and 12. The study protocol was approved by the Institutional Review Board of SYSUCC (B2015-00501). All the patients have provided written informed consent.

DNA extraction. For fresh-frozen samples (discovery cohort), genomic DNA from tumors and matched normal samples were isolated using the QIAamp DNA Mini Kit (Qiagen), according to the manufacturer's instructions. And for FFPE tumors (validation cohort), DNA was extracted using QIAamp DNA FFPE Tissue Kit (Qiagen). All DNA was quantified using the Qubit Fluorometer, and the quality of DNA was tested using agarose gel electrophoresis.

Whole-exome sequencing. To construct whole-exome capture libraries, $2 \mu \mathrm{g}$ of genomic DNA form each fresh-frozen tumor and matched normal sample was randomly fragmented by Covaris into $200 \sim 250 \mathrm{bp}$. After fragmentation, these fragments were purified and ligated by BGI-designed PE Index Adaptors, then captured with the BGI-Exome-V4 kit ( 59 Mb; BGI, Shenzhen, China). All the constructed libraries were loaded on Hiseq4000 platform (Illumina) and the sequences were generated as 150 -bp paired-end reads.

Sequencing reads which contained sequencing adapters, more than $10 \%$ of unknown bases and low-quality bases $(>50 \%$ bases with base quality $<5)$ were removed. Processed sequencing reads were then aligned to UCSC human reference genome (hg19) using BWA-MEM (v0.7.12). Picard (v1.84, http://broadinstitute. github.io/picard/) was used to generate chromosomal coordinate-sorted bam files and to remove PCR duplications. Then we performed base quality score recalibration and local realignment of the aligned reads using the Genome Analysis Toolkit (GATK v3.4) ${ }^{55}$ to improve alignment accuracy.

Somatic mutation detection. After sequencing data processed, the potential somatic substitutions (SNVs) were called by MuTect (v1.1.7) ${ }^{56}$ with default parameter based on paired alignment files (tumor and matched normal). Somatic InDels were predicted with GATK SomaticIndelDetector with default parameters In this process, the following SNVs were eliminated: (i) mutations reported in dbSNP (v142) with mutated allele supporting reads $<10$; (ii) distance between two mutations is $<3$; (iii) strand bias (either plus or minus strand supporting reads divided by total variant supporting reads) is $>0.9$; (iv) mutations reported in 1000 Genome Project April 2015 release; the National Heart, Lung, and Blood Institute (NHLBI) Grand Opportunity (GO) Exome Sequencing Project (ESP) ESP6500SIV2 release and The Exome Aggregation Consortium (ExAC) database release 0.3 with a frequency of $>0.01$. For somatic InDels, mutations were further removed if the supporting reads from both tumor and normal samples was $<5$, median $/ \mathrm{mad}$ of InDel offsets from the starts or ends of the reads $\geq 5 \mathrm{bp}$, average mapping qualities of the reads supported reference and InDel in tumor samples was $\leq 20$, or in simple repeat regions. All SNVs and InDels were subsequently annotated by ANNOVAR.

Targeted sequencing. To determine the mutations of candidate genes, we performed target deep sequencing on 114 carefully selected genes (Supplementary Fig. 7a). Genes were selected with the following criteria:

(a) Genes mutated in at least two of the 30 WES patients. These genes will be manually checked with literatures by two researchers in our institute to ensure the association with cancer. In addition, we also included genes mutated in one patient only if it was presented in the cancer gene census (CGC) database of COSMIC (https://cancer.sanger.ac.uk/census).

(b) Genes frequently mutated in $\mathrm{NPC}^{30}$, NKTCL ${ }^{47}$, LUAD (TCGA) ${ }^{31}$, LUSC (TCGA) ${ }^{32}$, and SCLC 57 . In addition, the top 20 frequently mutated genes provided by the Cancer Browse tools in COSMIC database (https://cancer. sanger.ac.uk/cosmic) for LUSC, LUAD, and SCLC were also considered. These genes were also manually checked as described in a).

(c) We also included NF-kB pathway genes that mutated in only one out of the 30 WES patients or frequently mutated in other cancers.

We then merged and removed duplicates for all genes. Thus, we have three categories of unique genes included in the 114 gene panel: cancer associated genes frequently mutated in pulmonary LELC or other related cancers, and NF-kB pathway genes. A list of genes for each category was shown in Supplementary 
Fig. 7a. And a customized DNA enrichment kit, capturing all exons from these 114 genes and targeting $\sim 700 \mathrm{k}$ genomic regions, was designed. Genomic DNA (200-500 ng) from each sample for validation was used for hybrid capture and library construction. Libraries were then sequenced on Hiseq4000 platform (Illumina) with $2 \times 150$ bp paired-end reads. Sequenced reads were processed as WES data described above.

We validated the somatic mutations identified in WES by observing at least three reads supporting the mutant allele in the target deep sequencing. In addition, the Pearson correlation coefficient was calculated to estimate the consistency of mutation frequency identified in WES and TDS.

An in-house pipeline was employed to detect SNVs for FFPE samples that do not have matched normal controls. First, we examined all candidate mutation sites by excluding those: (i) alignment quality $<20$ and sequencing base quality $<20$; (ii) sequencing depth $<10$ and mutant allele supporting reads $<4$; (iii) mutation frequency $<5 \%$ or between 45 and 55\%; (iv) registered in dbSNP142, ExAC, ESP6500, and the 1000 Genomes project with a frequency of $>0.01$ unless they were registered in the COSMIC; (v) presented in our SNP database constructed from the 30 normal samples in discovery cohort; (vi) distance between two mutations $<3$ or strand bias $>0.9$. We tested the pipeline on 30 tumors which had both WES and TDS data and achieved an overall validation rate of $82 \%$.

Mutational signature analysis. Mutational signature was first identified using the BayesNMF algorithms. Firstly, the count of somatic mutations was calculated for each type of substitution (96 trinucleotide mutation contexts) to generate the mutational catalogue. Then we ran the Bayesian NMF 1000 times with the hyperparameter for the inverse gamma prior setting to $10(a=10)$; the iterations were terminated when the tolerance for convergence was $<10-7$; and half-normal was chose as 'pirors' for this algorithm. We identified two significant signatures in our data set. To increase the confidence of the findings, we also used the NMF methodology described by Alexandrov et al. ${ }^{10}$ (http://www.mathworks.com/ matlabcentral/fileexchange/38724). The number of cycles of NMF runs was set from 1 to 15 , and 2 was the best estimation due to the high stability and low reconstruction error. The two signatures were then compared to the known mutational processes from the COSMIC signature database (http://cancer.sanger. ac.uk/cosmic/signatures) by calculating the cosine similarity as following: ${ }^{58}$

$$
\operatorname{sim}(A, B)=\frac{\sum_{K=1}^{K} A_{K} B_{K}}{\sqrt{\sum_{K=1}^{K}\left(A_{K}\right)^{2}} \sqrt{\sum_{K=1}^{K}\left(B_{K}\right)^{2}}}
$$

where $K$ is the number of mutation types $(K=96)$. Because the elements of $A$ and $B$ are nonnegative, the cosine similarity has a range between 0 and 1 . When the cosine similarity is 1 between two signatures, these signatures are exactly the same. In contrast, when the similarity is 0 , the signatures are independent.

Copy number analysis. Analysis of copy number alterations was performed on the basis of DNA profiling of each tumor or normal sample on Affymetrix OncoScan ${ }^{\circledR}$ CNV FFPE Assay. Genomic DNA was quantified using a Qubit ${ }^{\mathrm{m}}$ Fluorometer and at least $80 \mathrm{ng}$ of genomic DNA with DNA concentration $\geq 12 \mathrm{ng} / \mu \mathrm{l}$ was required for each sample. Before processing according to the OncoScan ${ }^{\circledR}$ CNV FFPE Assay Kit protocol, DNA integrity was verified by agarose gel electrophoresis. The raw intensity data (DAT) were analyzed with Affymetrix ${ }^{\circledast}$ GeneChip $^{\circledast}$ Command Console $^{\circledast}$ (AGCC) software (v4.1.2; Affymetrix) and generated array fluorescence intensity (CEL) files. For FFPE and frozen samples, normalized log R ratio (LRR) and $\mathrm{B}$ allele frequency (BAF) for all the available probes in each sample were extracted by the OncoScan Console (v1.2; Affymetrix) from fluorescence intensity (CEL) files using FFPE Analysis NA33 and REF103 Analysis NA33 as normal reference panel, respectively. Samples were excluded from downstream analysis if single-nucleotide polymorphism quality control (SNPQC) $\leq 20$ or the median absolute value pairwise difference $(M A P D) \geq 0.3$. Segments were then detected by Nexus Express software using the TuScan segmentation algorithm with default parameters. We removed segments that spanned $<100 \mathrm{~kb}$ or contained $<25$ probes. Broad and focal CNVs were identified using GISTIC2.0 algorithm ${ }^{28}$ with parameters: -genegistic 1 -broad 1 -brlen 0.98 -conf 0.95 -armpeel 1 -js 8 . We also removed regions corresponding to germline copy-number alterations by applying filters generated from the TCGA and our normal samples when performing GISTIC analysis.

Pathway enrichment analysis. We performed pathway enrichment analysis by integrating somatic mutation and SCNA data using both Gene Set Enrichment Analysis (GSEA, http://software.broadinstitute.org/gsea) and DAVID (https:// david-d.ncifcrf.gov/). GSEA pathway analysis was based on the Kyoto Encyclopedia of Genes and Genomes (KEGG) database from MSigDB version 6.0. The significance enrichment pathways were determined by a hypergeometric test and the FDR $q$-values was $<0.05$.

Comparison analysis. We downloaded the SNV/InDels and CNV data of LUAD, LUSC, STAD, HNSC from The Cancer Genome Atlas (TCGA, http://gdac. broadinstitute.org/). Mutation data of NPC (Lin et al. $)^{30}$, NKTCL (Jiang et al. $)^{47}$, SCLC (George et al. $)^{57}$ were adopted from the latest publications.
Total somatic mutation rate for each cancer type was calculated with the nonsilent mutations and tested by Student's $t$-test. The association between pulmonary LELC and other cancer types of six base substitutions was calculated by Pearson correlation coefficient. Hierarchical clustering of 96 possible mutation types (Six base substitutions each with 16 possible combinations of neighboring bases) was performed by the 'aheatmap' function with euclidean distance and 'ward.D2' agglomeration method of R package NMF (https://cran.r-project.org/web/ packages/NMF/index.html). We also generated a phylogenetic tree by first computing the Pearson correlation between all cancers and using these dissimilarity values to cluster the cancers.

Broad and focal SCNAs were analyzed with GISTIC 2.0 algorithm for each cancer type. The thresholds for gene copy number alterations were: amplifications, GISTIC score $=2$; gains, GISTIC score $=1$; losses, GISTIC score $=-1$; deletions, $=-2$. The landscape and frequency of copy number alterations was displayed on The Integrative Genomics Viewer (IGV, v 2.2.7).

For EBV abundance analysis, sequencing reads that could not mapped to the human reference genome(hg19) were extracted and realigned to the EBV genome type 1 (NC_007605.1) by BWA-MEM (v0.7.12). Then the number of mapped reads was calculated to estimate the EBV abundance among all the whole-exomesequenced samples.

Immunohistochemical staining. The expression of LMP1, PD-L1, and CD8 was determined in FFPE pulmonary LELC sections by immunohistochemical staining After de-waxing, the sections were subjected to antigen retrieval and staining in the automated slide processing system BenchMark XT (Ventana Medical systems Inc., Tucson, AZ) with the OptiView Amplification kit (Ventana Medical Systems Inc.). The primary antibody used in this study was anti-LMP1 mouse monoclonal antibody (CS.1-4, Dako), anti-PD-L1 rabbit monoclonal antibody (E1L3N, Cell Signaling Technology) and anti-CD8 mouse monoclonal antibody (4B11, Leica Microsystems). The LMP1 and PD-L1 expression was assessed by two independent pathologists by assigning a proportion score and an intensity score (0, absent; 1 , weak; 2 , moderate; and 3 , strong). The $\mathrm{H}$-score was the product of proportion multiplied by intensity scores, ranging from 0 to 300 . According to the report by Yvonne Y. Li et al., the LMP1 expression was categorized into absence/low (score $0-100$ ) and high (score 101-300) ${ }^{26}$. According to International TILs Working Group 2014, we scored CD8 + TILs as a percentage of positive staining in the stromal areas alone, with areas occupied by carcinoma cells excluded ${ }^{59}$.

Cell culture. Immortalized human lung bronchial epithelial cell line (Beas-2B) were generously provided by Prof. Liang Chen (Jinan University, Guangzhou, China $)^{60}$. All the cell lines were cultured in DMEM supplemented with $10 \%$ fetal bovine serum and antibiotics $(10,000 \mathrm{U} / \mathrm{mL}$ penicillin and $10 \mathrm{mg} / \mathrm{mL}$ streptomycin). All the cells were maintained in a humidified incubator at $37^{\circ} \mathrm{C}$ with $5 \% \mathrm{CO}_{2}$

Knockdown of endogenous TRAF3. The sequences of the TRAF3 siRNA were designed by RIBOBIO (Guangzhou, China) as following: sense, $\left(5^{\prime}-3^{\prime}\right) 5^{\prime}$-GGAA GAUUCGCGACUACAAdTdT- $3^{\prime}$ and antisense, $3^{\prime}$-dTdTCCUUCUAAGCGCUG AUGUU-5'. Recombinant lentivirus expressing either vector (GV248) or GV248 subcloned with TRAF3 siRNA was constructed by GENE Corporation (Shanghai, China). According to the manufacturer's instructions, lenti-shTRAF3 (shTRAF3) and negative control (shNC) with package vectors were transfected into HEK-293 T cells for $72 \mathrm{~h}$. Lentivirus supernatants were harvested and used to infect BEAS-2B cells with $2 \mu \mathrm{g} / \mathrm{ml}$ polybrene for $48 \mathrm{~h}$. The cells were cultured with $2 \mu \mathrm{g} /$ $\mathrm{ml}$ puromycin (Thermofish Scientific) in the medium for 7 days to construct TRAF3 down-regulated cells (BEAS-2B- shTRAF3), as well as negative control cells BEAS-2B- shNC.

Migration assay. Beas-2B cells were seeded onto transwell inserts (Corning, 3422) in 24-well plates and incubated for $48 \mathrm{~h}$. The inserts were washed with PBS, and non-migrating cells were wiped off from the top side. Migrated cells were fixed with $4 \%$ paraformaldehyde and stained with $0.1 \%$ crystal violet solution, and nuclei were counted.

Colony formation assay. Beas-2B cells were plated on 6-well plates with a density of 300 cells per well in triplicate. After 2 weeks, the cells were fixed with $4 \%$ polyformaldehyde, and then stained with freshly prepared diluted $0.1 \%$ crystal violet solution for $20 \mathrm{~min}$. After rinsing with distilled water, colonies of 50 or more cells were counted under a stereomicroscope.

Short-term cell proliferation assays. Beas-2B cells were seeded on the 96-well plates at optimized confluence in triplicate and were grown for a total of 4 days. 3(4, 5-dimethylthiazol-2-yl) - 2, 5-diphenyltetrazolium bromide (MTT) incorporation was performed to quantify the number of cells according to the manufacturer's guidance (Thermofish Scientific).

Western blotting. The whole-cell lysates were prepared with extraction buffer $(50$ $\mathrm{mM}$ Tris- $\mathrm{HCl}, \mathrm{pH} 7.4,150 \mathrm{mM} \mathrm{NaCl}$ and $0.5 \%$ Nonidet P-40) supplemented with complete protease and phosphatase inhibitor cocktail (Roche). The procedures for 
standard western blotting were performed according to the manufacturer's guidance. The antibodies specific for TRAF3 (Cat\#: 4729, 1:1000 dilution), P100 (Cat\#, 4882, 1:1000 dilution), P52 (Cat\#, 4882, 1:1000 dilution), IK $\beta a$ (Cat\#, 2859, 1:1000 dilution), and $\beta$-actin (Cat\#, 4970, 1:1000 dilution) were purchased from Cell Signaling Technologies, USA. The uncropped and unprocessed scans of Fig. $4 \mathrm{c}$ are shown in Supplementary Fig. 16.

Statistical analysis. Two-tailed Student's $t$-test and Fisher's exact test were used for continuous and discrete variables, respectively. Pearson's chi-squared test was used for comparison of response rate difference. And survival probability and difference were analyzed using log-rank test and a Cox proportional hazards model (multi-variate analysis). All statistical analysis was done with standard R packages. A two-sided $p$-value of $<0.05$ defined statistical significance.

Reporting summary. Further information on research design is available in the Nature Research Reporting Summary linked to this article.

\section{Data availability}

Patient clinical data (deidentified) were provided in the Supplementary Data 1 and 12. The complete somatic mutation calls can be found in Supplementary Data 4, 5 and 6 . The VCF of exome sequencing and targeted sequencing that support this study have been deposited both in the European Variation Archive (EVA) at the EMBL-EBI under accession number PRJEB32689 and the CNGB (China National GeneBank) Nucleotide Sequence Archive (CNSA) database under the accession codes CNP0000327 (https://db. cngb.org/cnsa/). All the other data supporting the findings of this study are available within the article and its Supplementary Information Files and from the corresponding authors upon reasonable request.

Received: 31 March 2018 Accepted: 10 June 2019

Published online: 16 July 2019

\section{References}

1. Hayashi, T. et al. Cytopathologic features and differential diagnostic considerations of primary lymphoepithelioma-like carcinoma of the lung. Diagn. Cytopathol. 40, 820-825 (2012).

2. Begin, L. R., Eskandari, J., Joncas, J. \& Panasci, L. Epstein-Barr virus related lymphoepithelioma-like carcinoma of lung. J. Surg. Oncol. 36, 280-283 (1987).

3. Han, A. J., Xiong, M. \& Zong, Y. S. Association of Epstein-Barr virus with lymphoepithelioma-like carcinoma of the lung in southern China. Am. J. Clin. Pathol. 114, 220-226 (2000).

4. Travis, W. D. et al. The 2015 World Health Organization Classification of lung tumors: impact of genetic, clinical and radiologic advances since the 2004 classification. J. Thorac. Oncol. 10, 1243-1260 (2015).

5. Irie, H. Y., Jaklitsch, M. T., Shaffer, K., Weinstein, M. \& Salgia, R. Unusual problems in breast cancer and a rare lung cancer case. Case 4. Primary lymphoepithelioma-like carcinoma of the lung. J. Clin. Oncol. 21, 2220-2222 (2003).

6. Fang, W. et al. PD-L1 is remarkably over-expressed in EBV-associated pulmonary lymphoepithelioma-like carcinoma and related to poor diseasefree survival. Oncotarget 6, 33019-33032 (2015).

7. Reck, M. et al. Pembrolizumab versus chemotherapy for PD-L1-positive nonsmall-cell lung cancer. N. Engl. J. Med 375, 1823-1833 (2016).

8. Gandhi, L. et al. Pembrolizumab plus chemotherapy in metastatic non-smallcell lung cancer. N. Engl. J. Med 378, 2078-2092 (2018).

9. Zhou, Y. et al. Immune-checkpoint inhibitor plus chemotherapy versus conventional chemotherapy for first-line treatment in advanced non-small cell lung carcinoma: a systematic review and meta-analysis. J. Immunother. Cancer 6, 155 (2018)

10. Alexandrov, L. B. et al. Signatures of mutational processes in human cancer. Nature 500, 415-421 (2013).

11. Vieira, V. C. \& Soares, M. A. The role of cytidine deaminases on innate immune responses against human viral infections. Biomed. Res. Int. 2013, 683095 (2013)

12. Stavrou, S. \& Ross, S. R. APOBEC3 proteins in viral immunity. J. Immunol 195, 4565-4570 (2015).

13. Roberts, S. A. et al. An APOBEC cytidine deaminase mutagenesis pattern is widespread in human cancers. Nat. Genet. 45, 970-976 (2013).

14. Hua, X. et al. MEGSA: a powerful and flexible framework for analyzing mutual exclusivity of tumor mutations. Am. J. Hum. Genet. 98, 442-455 (2016).

15. Chang, Y. L., Wu, C. T., Shih, J. Y. \& Lee, Y. C. Unique p53 and epidermal growth factor receptor gene mutation status in 46 pulmonary lymphoepithelioma-like carcinomas. Cancer Sci. 102, 282-287 (2011).
16. Huang, X., Ji, G., Wu, Y., Wan, B. \& Yu, L. LAMA4, highly expressed in human hepatocellular carcinoma from Chinese patients, is a novel marker of tumor invasion and metastasis. J. Cancer Res. Clin. Oncol. 134, 705-714 (2008).

17. Nagato, S. et al. Downregulation of laminin alpha4 chain expression inhibits glioma invasion in vitro and in vivo. Int. J. Cancer 117, 41-50 (2005).

18. Ho, T. H. et al. Differential gene expression profiling of matched primary renal cell carcinoma and metastases reveals upregulation of extracellular matrix genes. Ann. Oncol. 28, 604-610 (2017).

19. Govaere, O. et al. The PDGFRalpha-laminin B1-keratin 19 cascade drives tumor progression at the invasive front of human hepatocellular carcinoma. Oncogene 36, 6605-6616 (2017).

20. Liu, Q. et al. Lack of epidermal growth factor receptor gene mutations in exons 19 and 21 in primary lymphoepithelioma-like carcinoma of the lung. Thorac. Cancer 5, 63-67 (2014).

21. Wang, L. et al. Detection of rearrangement of anaplastic lymphoma kinase (ALK) and mutation of epidermal growth factor receptor (EGFR) in primary pulmonary lymphoepithelioma-like carcinoma. J. Thorac. Dis. 7, 1556-1562 (2015).

22. Tam, I. Y. et al. Distinct epidermal growth factor receptor and KRAS mutation patterns in non-small cell lung cancer patients with different tobacco exposure and clinicopathologic features. Clin. Cancer Res. 12, 1647-1653 (2006).

23. Chang, Y. L., Yang, C. Y., Lin, M. W., Wu, C. T. \& Yang, P. C. PD-L1 is highly expressed in lung lymphoepithelioma-like carcinoma: a potential rationale for immunotherapy. Lung Cancer 88, 254-259 (2015).

24. Lee, G. D. et al. MET exon 14 skipping mutations in lung adenocarcinoma: clinicopathologic implications and prognostic values. J. Thorac. Oncol. 12, 1233-1246 (2017)

25. Frampton, G. M. et al. Activation of MET via diverse exon 14 splicing alterations occurs in multiple tumor types and confers clinical sensitivity to MET inhibitors. Cancer Discov. 5, 850-859 (2015).

26. Li, Y. Y. et al. Exome and genome sequencing of nasopharynx cancer identifies NF-kappaB pathway activating mutations. Nat. Commun. 8, 14121 (2017).

27. Bruce, J. P., Yip, K., Bratman, S. V., Ito, E. \& Liu, F. F. Nasopharyngeal cancer: molecular landscape. J. Clin. Oncol. 33, 3346-3355 (2015).

28. Mermel, C. H. et al. GISTIC2.0 facilitates sensitive and confident localization of the targets of focal somatic copy-number alteration in human cancers. Genome Biol. 12, R41 (2011).

29. Cancer Genome Atlas Research Network. Comprehensive molecular characterization of gastric adenocarcinoma. Nature 513, 202-209 (2014).

30. Lin, D. C. et al. The genomic landscape of nasopharyngeal carcinoma. Nat. Genet. 46, 866-871 (2014).

31. Cancer Genome Atlas Research Network. Comprehensive molecular profiling of lung adenocarcinoma. Nature 511, 543-550 (2014).

32. Cancer Genome Atlas Research Network. Comprehensive genomic characterization of squamous cell lung cancers. Nature 489, 519-525 (2012)

33. Garcia-Sastre, A. \& Biron, C. A. Type 1 interferons and the virus-host relationship: a lesson in detente. Science 312, 879-882 (2006).

34. Chikuma, S., Kanamori, M., Mise-Omata, S. \& Yoshimura, A. Suppressors of cytokine signaling: potential immune checkpoint molecules for cancer immunotherapy. Cancer Sci. 108, 574-580 (2017).

35. Veeriah, S. et al. The tyrosine phosphatase PTPRD is a tumor suppressor that is frequently inactivated and mutated in glioblastoma and other human cancers. Proc. Natl Acad. Sci. USA. 106, 9435-9440 (2009).

36. Platanias, L. C. Mechanisms of type-I- and type-II-interferon-mediated signalling. Nat. Rev. Immunol. 5, 375-386 (2005).

37. Hajek, M. et al. TRAF3/CYLD mutations identify a distinct subset of human papillomavirus-associated head and neck squamous cell carcinoma. Cancer 123, 1778-1790 (2017)

38. Inoue, J. et al. Tumor necrosis factor receptor-associated factor (TRAF) family: adapter proteins that mediate cytokine signaling. Exp. Cell Res. 254, 14-24 (2000).

39. Bertin, J. et al. CARD11 and CARD14 are novel caspase recruitment domain (CARD)/membrane-associated guanylate kinase (MAGUK) family members that interact with BCL10 and activate NF-kappa B. J. Biol. Chem. 276, 11877-11882 (2001)

40. Jiang, C. \& Lin, X. Regulation of NF-kappaB by the CARD proteins. Immunol. Rev. 246, 141-153 (2012).

41. $\mathrm{Wu}, \mathrm{S}$. et al. LMP1 protein from the Epstein-Barr virus is a structural CD40 decoy in B lymphocytes for binding to TRAF3. J. Biol. Chem. 280, 33620-33626 (2005)

42. Wang, Z. et al. STAT3 activation induced by Epstein-Barr virus latent membrane protein 1 causes vascular endothelial growth factor expression and cellular invasiveness via JAK3 And ERK signaling. Eur. J. Cancer 46 2996-3006 (2010).

43. Li, L. et al. Viral oncoprotein LMP1 disrupts p53-induced cell cycle arrest and apoptosis through modulating K63-linked ubiquitination of p53. Cell Cycle 11, 2327-2336 (2012). 
44. Hariwiyanto, B., Sastrowiyoto, S., Mubarika, S. \& Salugu, M. LMP1 and LMP2 may be prognostic factors for outcome of therapy in nasopharyngeal cancers in Indonesia. Asian Pac. J. Cancer Prev. 11, 763-766 (2010).

45. He, J. Q., Saha, S. K., Kang, J. R., Zarnegar, B. \& Cheng, G. Specificity of TRAF3 in its negative regulation of the noncanonical NF-kappa B pathway. J. Biol. Chem. 282, 3688-3694 (2007).

46. Siu, L. L., Wong, K. F., Chan, J. K. \& Kwong, Y. L. Comparative genomic hybridization analysis of natural killer cell lymphoma/leukemia. Recognition of consistent patterns of genetic alterations. Am. J. Pathol. 155, 1419-1425 (1999).

47. Jiang, L. et al. Exome sequencing identifies somatic mutations of DDX3X in natural killer/T-cell lymphoma. Nat. Genet. 47, 1061-1066 (2015).

48. Zhang, L. et al. Gemcitabine plus cisplatin versus fluorouracil plus cisplatin in recurrent or metastatic nasopharyngeal carcinoma: a multicentre, randomised, open-label, phase 3 trial. Lancet 388, 1883-1892 (2016).

49. Zhang, Y. et al. Open-label, single-arm phase II study of pemetrexed in the treatment of patients with recurrent or metastatic nasopharyngeal carcinoma who have had prior platinum-based chemotherapy. Cancer Chemother. Pharmacol. 70, 611-615 (2012).

50. Yau, T. K. et al. A phase II study of pemetrexed combined with cisplatin in patients with recurrent or metastatic nanopharyngeal carcinoma. Oral. Oncol. 48, 441-444 (2012).

51. Santoro, M. G., Rossi, A. \& Amici, C. NF-kappaB and virus infection: who controls whom. EMBO J. 22, 2552-2560 (2003).

52. Hervas-Stubbs, S. et al. Direct effects of type I interferons on cells of the immune system. Clin. Cancer Res. 17, 2619-2627 (2011).

53. Ivashkiv, L. B. \& Donlin, L. T. Regulation of type I interferon responses. Nat. Rev. Immunol. 14, 36-49 (2014).

54. Jennings, R. N., Grayson, J. M. \& Barton, E. S. Type I interferon signaling enhances CD8 $+\mathrm{T}$ cell effector function and differentiation during murine gammaherpesvirus 68 infection. J. Virol. 88, 14040-14049 (2014).

55. DePristo, M. A. et al. A framework for variation discovery and genotyping using next-generation DNA sequencing data. Nat. Genet 43, 491-498 (2011).

56. Cibulskis, K. et al. Sensitive detection of somatic point mutations in impure and heterogeneous cancer samples. Nat. Biotechnol. 31, 213-219 (2013).

57. George, J. et al. Comprehensive genomic profiles of small cell lung cancer. Nature 524, 47-53 (2015).

58. Alexandrov, L. B., Nik-Zainal, S., Wedge, D. C., Campbell, P. J. \& Stratton, M. R. Deciphering signatures of mutational processes operative in human cancer. Cell Rep. 3, 246-259 (2013).

59. Salgado, R. et al. The evaluation of tumor-infiltrating lymphocytes (TILs) in breast cancer: recommendations by an International TILs Working Group 2014. Ann. Oncol. 26, 259-271 (2015).

60. Hong, S. et al. Upregulation of PD-L1 by EML4-ALK fusion protein mediates the immune escape in ALK positive NSCLC: implication for optional anti-PD1/PD-L1 immune therapy for ALK-TKIs sensitive and resistant NSCLC patients. Oncoimmunology 5, e1094598 (2016).

\section{Acknowledgements}

We thank Prof. Liang Chen (Jinan University, Guangzhou, China) for providing Beas-2B. We appreciate Yidu Cloud Corporation (Beijing, China) for management of clinical data. Finally, we would like to thank the patients of Sun Yat-sen University Cancer Center who contributed to this study. We acknowledge funding support from the National Key R\&D Program of China (2016YFC0905500, 2016YFC0905503); National Natural Science Funds of China (Grant number: 81602005, 81702283, 81872499, and 81602011); the Outstanding Young Talents Program of Sun Yat-sen University Cancer Center (16zxyc04); the Central Basic Scientific Research Fund for Colleges-Young Teacher Training Program of Sun Yat-sen University (17ykpy81); Science and Technology Program of Guangdong Province (2017B020227001); the Science, Technology and Innovation Commission of Shenzhen Municipality under grant (No.

JCYJ20160531193931852, JCYJ20170817145454378), Guangdong Enterprise Key Laboratory of Human Disease Genomics (2011A060906007), and Science and Technology Program of Yunnan Province (2016RA037). S.D.H is the winner of 2016 ASCO Conquer Cancer Foundation Merit Award for this study. S.D.H also recieve support from the Sun Yat-sen University Cancer Center Promotion Program for Talent Youth of National Natural Science Funds of China. The funding sources had no role in the design and conduction of the study; collection, management, analysis, and interpretation of the data; preparation, review, or approval of the manuscript; and decision to submit the manuscript for publication.

\section{Author contributions}

L.Z., S.D.H, K.W. and D.B.L. designed the study. S.D.H, D.B.L. and S.Z.L. performed medical record review, analyzed data, and prepared tables and figures. D.B.L. and S.Z.L performed WES, TDS, and SNP array analysis and prepared tables and figures. J.H.Z. performed cell-line experiments and prepared figures with assistance from W.F.F.; S.F. performed immunohistochemistry and ISH analysis and carried out pathological assessments. S.D.H., D.B.L. and S.Z.L. wrote the manuscript. W.F.F., Z.J.H., F.S., Z.Y.X., W.X., Z.H.Q., C.X., C.G., Z.Z.H., Z.Q.F., L.X.B., C.J.H., L.X.M., L.M.Y., Y.C., W.J., Y.H. M., X.X., Z.S.D., Y.Y.P., Z.Y.Y., Z.N.N., Z.H.Y., H.Y. and Z.L.; J.C. and J.W. contributed to drafting and revising the manuscript.

\section{Additional information}

Supplementary Information accompanies this paper at https://doi.org/10.1038/s41467019-10902-w.

Competing interests: The authors declare no competing interests.

Reprints and permission information is available online at http://npg.nature.com/ reprintsandpermissions/

Peer review information: Nature Communications thanks the anonymous reviewers for their contribution to the peer review of this work. Peer reviewer reports are available.

Publisher's note: Springer Nature remains neutral with regard to jurisdictional claims in published maps and institutional affiliations.

(c) Open Access This article is licensed under a Creative Commons Attribution 4.0 International License, which permits use, sharing, adaptation, distribution and reproduction in any medium or format, as long as you give appropriate credit to the original author(s) and the source, provide a link to the Creative Commons license, and indicate if changes were made. The images or other third party material in this article are included in the article's Creative Commons license, unless indicated otherwise in a credit line to the material. If material is not included in the article's Creative Commons license and your intended use is not permitted by statutory regulation or exceeds the permitted use, you will need to obtain permission directly from the copyright holder. To view a copy of this license, visit http://creativecommons.org/ licenses/by/4.0/.

(C) The Author(s) 2019 